\title{
Corrupción en la gestión de recursos públicos asignados en la emergencia sanitaria COVID-19 en el gobierno regional de La Libertad - Perú 2020
}

Corruption in the management of public resources allocated in the health emergency COVID-19 in the regional government of La Libertad - Peru 2020

\section{Johanna Jaritssielle, Zavaleta Davis ${ }^{\text {a }}$; johannahzd@ @mail.com \\ Orcid: https://orcid.org/0000-0002-2866-8411 \\ Leslye Anjy, Chávez Santivañez ${ }^{\text {b }}$, anjychavez79@gmail.com \\ Orcid: https://orcid.org/0000-0002-6643-6494}

${ }^{a}$ Escuela de Administración, Universidad Privada Antenor Orrego, La Libertad - Perú

${ }^{\mathrm{b}}$ Instituto de Innovación y Formación Científica, La Libertad - Perú

Recibido Abril/23/2020 • Aceptado: Junio/02/2020 • Publicado: Junio/30/2020

\section{RESUMEN}

La corrupción es un mal que la población ha venido arrastrando por mucho tiempo y con la presente investigación se pretende conocer hasta donde llego la corrupción en la gestión de los recursos públicos asignados en la emergencia sanitaria COVID-19 en el gobierno regional de La Libertad - Perú 2020, para lo cual se utilizó una metodología del tipo descriptiva con la técnica de recolección de datos en la cual se estudió los beneficios que le fueron entregados al gobierno regional de La Libertad en plena crisis sanitaria por parte del estado peruano, y se llegó a la conclusión que pese a las transferencias económicas y los subsidios monetarios que suponen un apoyo para los sectores más golpeados por los efectos de la pandemia, no se ha logrado llegar ni a un $70 \%$ de avance de la ejecución del presupuesto asignado a las entidades públicas del gobierno regional y local, debido a la intervención de las irregularidades que cometen los "líderes "defensores del bienestar público, en el direccionamiento de los fondos para beneficio propio.

Palabras clave: COVID-19, corrupción y gobierno regional La Libertad.

a Johanna Jaritssielle, Zavaleta-Davis y ${ }^{\mathrm{b}}$ Leslye Anjy, Chávez-Santivañez

Articulo Protegido por Licencia Creative Commons: BY-NC-ND / Protected by Creative Commons: BY-NC-ND.

Sendas es una revista de acceso abierto / Sendas is an Open Access Journal. 


\section{ABSTRACT}

The corruption is an evil that the population has been dragging on for a long time and with this investigation it is intended to know how far corruption has gone in the management of public resources allocated in the health emergency COVID-19 in the regional government of La Libertad - Peru 2020, for which a descriptive type methodology was used with the data collection technique in which the benefits that were delivered to the regional government of La Libertad in the midst of a health crisis by the Peruvian state were studied, and it was arrived at to the conclusion that despite economic transfers and monetary subsidies that provide support for the sectors hardest hit by the effects of the pandemic, it has not been possible to reach even $70 \%$ progress in the execution of the budget assigned to the entities public of the regional and local government, due to the intervention of the irregularities committed by the "leaders" defending public welfare, in the direction use of funds for personal gain.

Keywords: COVID-19, corruption and regional government La Libertad.

\section{Introducción}

La investigación comprende el ámbito de gestión pública que se viene desarrollando en el estado de emergencia sanitaria a nivel nacional, enfatizando la situación que presenta el departamento de La Libertad. Cuyo propósito recae en la exposición de las irregularidades de administración, a mano de las entidades públicas, en los recursos otorgados para el apoyo de los más desfavorecidos a causa del covid-19. Considerando las distintas propuestas de ayuda gubernamental a través de los programas sociales y subsidios estatales para la reactivación económica, que incluyen: "El bono familiar universal", "bono independiente", "bono rural", "reactiva" y "arranca Perú", por mencionar algunos. Además de las designaciones presupuestales otorgadas para la compra de alimentos de primera necesidad que serían entregados a través de "las canastas familiares" para los

\footnotetext{
a Johanna Jaritssielle, Zavaleta-Davis y ${ }^{\mathrm{b}}$ Leslye Anjy, Chávez-Santivañez Articulo Protegido por Licencia Creative Commons: BY-NC-ND / Protected by Creative Commons: BY-NC-ND. Sendas es una revista de acceso abierto / Sendas is an Open Access Journal.
} 
sectores más pobres, en las distintas localidades del país.

Las cuales son reconocidas por las cifras millonarias que abarcan y la distribución deficiente y oculta de las mismas, que supondrían el resguardo del beneficio propio de los altos mandos de las unidades públicas por medio de la supuesta malversación de fondos; lo que estaría privando de la ayuda a los verdaderos destinatarios, quienes padecen a los efectos de la pandemia.

Se pensó que la crisis financiera del 2008, con la caída de Lehman Brothers , escribió en la economía mundial una página de la que hoy muchos países no pueden pasar, sin embargo a fines 2019 se detectó una amenaza latente que se ha propuesto castigar la estabilidad mundial y que para muchos sería "la peor recesión desde la Segunda Guerra Mundial, y la primera vez desde 1870 en que tantas economías experimentarían una disminución del producto per cápita “(2020) Banco mundial. Pues con un total de 472216 fallecidos, 9100090 casos confirmados a nivel mundial (2020) trading view, se prevé una caída del PBI global en 5.2 para este año, según el Banco Mundial; además de un afección completa o parcial de los empleos, en la que 4 de cada 5 personas que constituyen la fuerza laboral mundial (81\%) se vieron perjudicadas (2020) informe de la OIT y una disminución generalizada de los precios de los productos básicos. (2020) banco mundial. Impulsando a la toma de medidas políticas tanto en salud como economía para contrarrestar los efectos de la pandemia.

En el Perú, el panorama no es diferente, con un total de 257.447 mil casos confirmados y 8.223 mil fallecidos al 22de junio del 2020, según el INS, los pronósticos no son alentadores.

De acuerdo al informe del banco mundial El Perú registraría una caída del PBI en $12 \%$ para este año, siendo la economía más afectada después de Belice con $13.5 \%$. Esta previsión ya se percibe con una disminución de $-3,4 \%$, solo en el primer trimestre de este año, que conllevó

${ }^{\mathrm{a}}$ Johanna Jaritssielle, Zavaleta-Davis $\mathrm{y}^{\mathrm{b}}$ Leslye Anjy, Chávez-Santivañez Articulo Protegido por Licencia Creative Commons: BY-NC-ND / Protected by Creative Commons: BY-NC-ND. Sendas es una revista de acceso abierto / Sendas is an Open Access Journal. 
a que 1216600 personas perdieran su empleo solo en la capital, tras acatar las normativas de aislamiento, el cierre de establecimientos comerciales y la restricción de acceso tanto de fronteras internacionales como nacionales, por la declaración de estado de emergencia sanitaria nacional por noventa días y un período posterior del mismo tiempo, afectando de sobremanera a las MYPES e incrementando la informalidad a niveles superiores a los alguna vez registrados.

La desesperación de la población peruana por la escasez de recursos, en especial el monetario, indujo a la aplicación de políticas económicas que promuevan la reactivación y preserven el bienestar público. Designando en primera instancia el decreto de urgencia que autoriza el retiro progresivo de hasta el $25 \%$ de AFP y la reforma del sistema de pensiones. Así como 4 subsidios monetarios otorgados para familias vulnerables, en los que se destaca Bono Yo me quedo en casa, dirigido a hogares que se encuentran en situación de pobreza y pobreza extrema a través de dos pagos de s/. 380 Cada uno; Bono Independiente, dirigido a los hogares de los trabajadores independientes en situación de vulnerabilidad; Bono Rural, Dirigido a los hogares vulnerables en las zonas rurales y Bono Familiar Universal dirigido a los hogares vulnerables que aún no han sido beneficiados con los demás bonos otorgados por el Estado mediante un único pago de s/ 760.

A lo que se sumaría las trasferencias económicas, a través del ministerio de Economía y finanzas, a las diversas instituciones públicas para la contratación directa de bienes y servicios con el fin de salvaguardar el bienestar de los ciudadanos. Sin embargo, para perjuicio de la comunidad, estos pronunciamientos habrían sido aprovechados para el incremento del patrimonio de los ejecutores públicos en los distintos ámbitos de gobierno, a través de malversación de estos fondos, trayendo consigo únicamente la exposición al riesgo de la salud de la población. Lo que

a Johanna Jaritssielle, Zavaleta-Davis y ${ }^{\mathrm{b}}$ Leslye Anjy, Chávez-Santivañez Articulo Protegido por Licencia Creative Commons: BY-NC-ND / Protected by Creative Commons: BY-NC-ND. Sendas es una revista de acceso abierto / Sendas is an Open Access Journal. 
quedaría demostrado a través de los distintos casos de corrupción a nivel nacional, con 264 denuncias ante las fiscalías especializadas en delitos de corrupción del Ministerio Público. Como expone el informe de la procuraduría pública (2020).

Los más destacados son las irregularidades en compras de implementos de bioseguridad, equipos médicos, productos de limpieza y aseo personal, servicios de alimentación y otros. Como es el caso del "Centro Nacional de Abastecimiento de Recursos Estratégicos en Salud (Cenares) el cual fue intervenido por presuntas irregularidades en la compra de 1'257,900 unidades de mascarillas N-95, destinadas a el personal de salud por el coronavirus. Donde, según la Contraloría, el total de dinero invertido era de 24 millones 654 mil 840 soles, por lo que deducen, se habría ahorrado más de 4 millones de soles" (2020) Gestión, cuyo paradero no ha sido registrado.

De modo similar se registraron inconsistencias en las contrataciones de la
Policía Nacional del Perú donde la suma de los productos adquiridos, presuntamente de manera irregular a través de empresas sin experiencia en el rubro y con nexos con policías en actividad, llegó a los S/800 mil soles, en Lima, pero esta situación se habría repetido en las distintas regiones del país (2020) Gestión.

Es así que la procuraduría informó que el $51 \%$ del total de casos se concentra en siete regiones, estas son: Áncash, Lima, Arequipa, La Libertad, Junín, Ayacucho y Cajamarca. Donde las dos primeras encabezan la lista con 72 casos cada una.

La libertad, por su parte, registró 50 casos, donde prevalece la investigación del general PNP Lucas Núñez Córdova, tras un proceso administrativo disciplinario por estar presuntamente involucrado en la compra irregular de productos de limpieza y aseo personal durante la emergencia sanitaria de la COVID-19, por un monto de 10 millones de soles. (2020) RPP.

Modalidad que se reitera en el caso de incautación de productos de aseo y

\footnotetext{
a Johanna Jaritssielle, Zavaleta-Davis y ${ }^{\mathrm{b}}$ Leslye Anjy, Chávez-Santivañez Articulo Protegido por Licencia Creative Commons: BY-NC-ND / Protected by Creative Commons: BY-NC-ND. Sendas es una revista de acceso abierto / Sendas is an Open Access Journal.
} 
limpieza sin registro sanitario; que habría sido distribuidos a los centros de salud de la provincia Sánchez Carrión, denotando elevaciones exorbitantes en los precios de compra y el atentado contra la salud pública al carecer de la autorización de la Dirección General de Medicamentos, Insumos y Drogas (DIGEMID); en base a la denuncia del director de la Red de Salud, Omar García (2020) RPP.

Situación que se agrava a medida que avanza el proceso de investigación de los 724 casos en trámite por presuntos delitos de corrupción, que se originaron en el marco de la pandemia, registrados por la PPEDC a nivel nacional desde el 11 de marzo hasta el 04 de junio del presente año.

Quiroz (2014) define la corrupción como el mal uso del poder político burocrático por parte de funcionarios coludidos con mezquinos intereses privados para obtener ventajas económicas o políticas contrarias a las metas del desarrollo social mediante la malversación o el desvío de recursos públicos y la distorsión de las políticas e instituciones.

Said (2013) define a la corrupción como el incumplimiento de una obligación institucional inherente al cargo por parte de un funcionario, para la obtención de un beneficio personal extra posicional, otorgado al funcionario por un particular o empresa que lo soborna, o al que el funcionario extorsiona; generalmente dirigido a obtener para el particular o empresa beneficios propios. Intenté poner en claro que la cuestión se centra en la obtención de beneficios personales por los funcionarios, quienes aprovechan el cargo que ejercen en la Administración Pública para "negociar" los espacios de ilicitud.

Sánchez (2012) señala que la corrupción adquiere distintos matices: abuso de poder, tráfico de influencias, compadrazgo, amiguismo, soborno, cohecho, mal uso de los conocimientos, fraude, aceptación de obsequios a cambio de favores, entre otros que forman parte de lo que denomina corrupción administrativa, que se distingue de la

\footnotetext{
a Johanna Jaritssielle, Zavaleta-Davis y ${ }^{\mathrm{b}}$ Leslye Anjy, Chávez-Santivañez Articulo Protegido por Licencia Creative Commons: BY-NC-ND / Protected by Creative Commons: BY-NC-ND. Sendas es una revista de acceso abierto / Sendas is an Open Access Journal.
} 
corrupción política porque la primera afecta principalmente a los burócratas que forman parte de la administración pública.

Para algunos, el problema de la corrupción es esencialmente moral, y las soluciones deben apuntar, por una parte, a corregir los mecanismos de selección y control de los funcionarios públicos, y por otra, a la educación moral cívica de gobernantes y gobernados.

Otros consideran que se trata de un problema político referido a la estructura de la organización político-administrativa del Estado, ya que concentra en los funcionarios superiores amplios poderes de decisión, con una gran cuota de discrecionalidad y escasa o nula transparencia y control.

\section{$\underline{\text { Metodología }}$}

En la presente investigación se utilizó el método descriptivo, de diseño simple con la técnica de consolidación de los datos para lo cual se usaron sus respectivos instrumentos.

\section{Resultados}

A pesar de las grandes inyecciones de flujo de dinero que se les brindo a los gobiernos regionales destinados para la compra de insumos y materiales necesarios para enfrentar el Covid 19, en general se ha registrado un avance del $67.7 \%$ en la inversión de efectivo, tal como lo muestra la gráfica 1 .

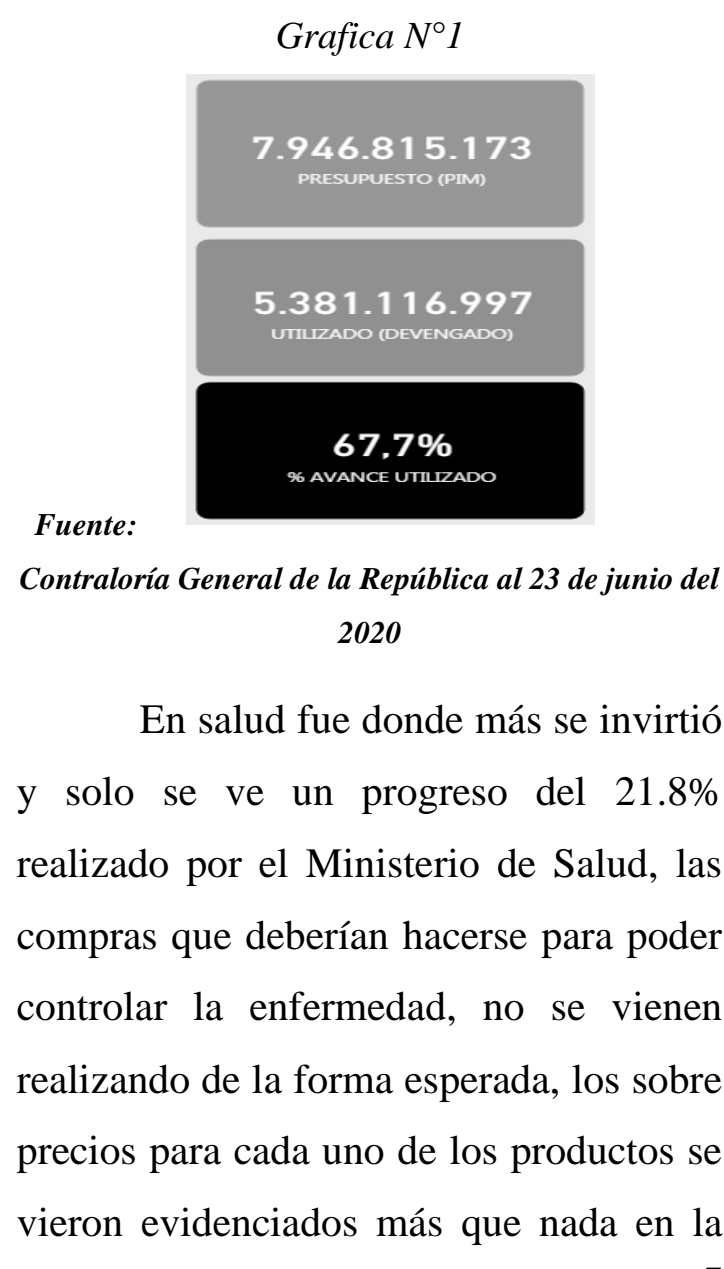

${ }^{\mathrm{a}}$ Johanna Jaritssielle, Zavaleta-Davis y ${ }^{\mathrm{b}}$ Leslye Anjy, Chávez-Santivañez Articulo Protegido por Licencia Creative Commons: BY-NC-ND / Protected by Creative Commons: BY-NC-ND. Sendas es una revista de acceso abierto / Sendas is an Open Access Journal. 
compra de las mascarillas que son de uso indispensable para este sector, un caso más concreto lo podemos encontrar en Chiclayo donde la fiscalía encontró que las compras de mascarillas, alcohol en gel y demás utensilios superaban el millón de soles y lo peor estos productos no eran adecuados para el uso humano.

En la gráfica 2 se compara el porcentaje de avance en la inversión del dinero designado que tienen los diversos sectores sociales que se vienen manejando durante esta crisis:

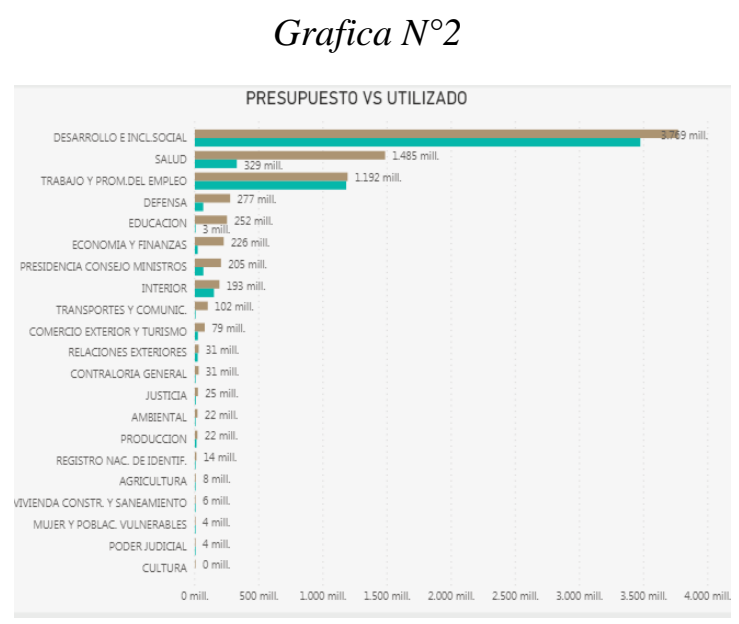

Fuente: Contraloría General de la República al 23 de junio del 2020

Por el lado de los beneficios sociales entregados a la población la contraloría General de la Republica reviso el listado de los beneficiarios de las canastas familiares en la cual se identificó que más de 100,000 funcionarios públicos habrían sido beneficiados con las canastas familiares que estaba destinada a ser de ayuda a las familias más vulnerables. Encontrando así solo en Lima 180 funcionarios, en Callao, Piura y Junín 136, 120 y 87 funcionarios, y son en estos lugares donde el Covid 19 afecto con mayor fuerza en el Perú.

En la gráfica 3 podemos observar el reporte oficial de las compras de la canasta familiar y el porcentaje de la entrega.

\section{Grafica $N^{\circ} 3$}

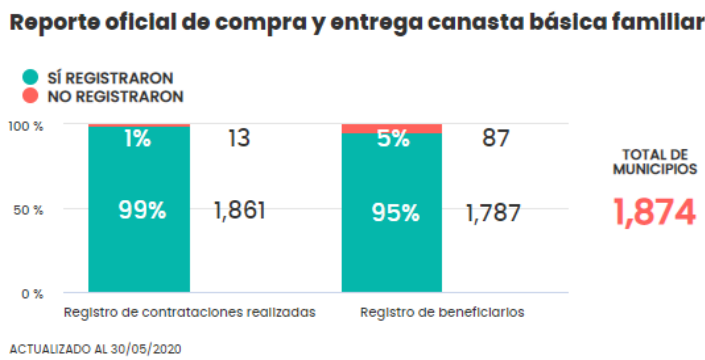

Fuente: Contraloría General de la República al 23 de junio del 2020

El bono de la canasta familiar era para beneficio directo hacia la población más necesita que no fue identificada en los

a Johanna Jaritssielle, Zavaleta-Davis y ${ }^{\mathrm{b}}$ Leslye Anjy, Chávez-Santivañez

Articulo Protegido por Licencia Creative Commons: BY-NC-ND / Protected by Creative Commons: BY-NC-ND.

Sendas es una revista de acceso abierto / Sendas is an Open Access Journal. 
bonos anteriores, las municipalidades tenían el deber de hacer llegar a estas familias una canasta que les permitirá sobrevivir esta cuarentena. Para poder lograr esto, se distribuyó poco más de 200 millones de soles y en la gráfica $\mathrm{N}^{\circ} 1$ podemos observar lo bien que se vino realizando este proyecto, donde solo para el 30 de mayo se necesitaba realizar un $1 \%$ de compras y beneficiar a un $5 \%$ de familias, el objetivo planteado parecía cumplido, pero en la gráfica $\mathrm{N}^{\circ} 4$ y grafica $\mathrm{N}^{\circ} 5$ nos damos cuenta que nada es como parece puesto que dentro de la lista de los más necesitados se encontraban funcionarios públicos que en esta pandemia aun contaban con un sueldo fijo y muchos de ellos se encontraban en poblaciones vulnerables.

\section{Grafica $N^{\circ} 4$}

\section{Funcionarios y servidores públicos que habrian recibido canastas básicas familiares}

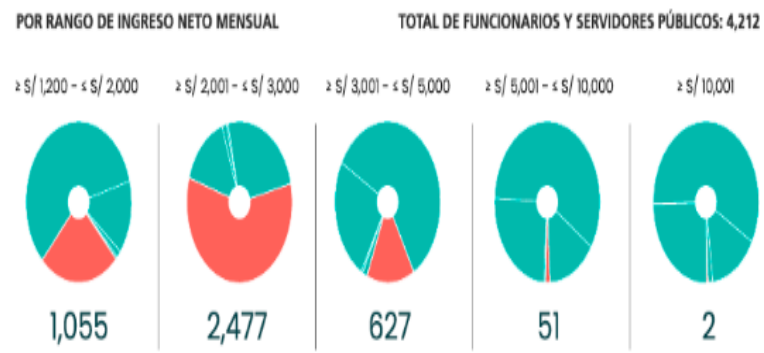

Fuente: Contraloría General de la República al 23 de junio del 2020

Grafica $N^{\circ} 5$

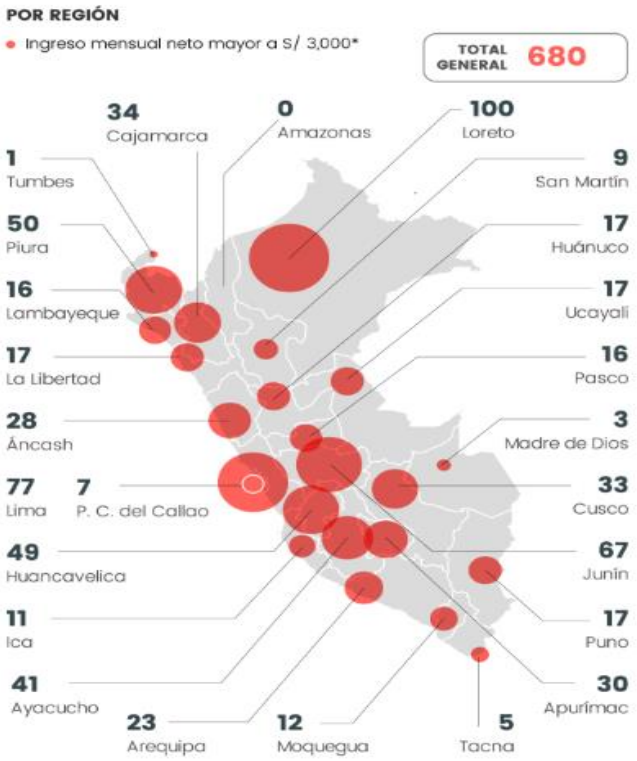

Fuente: Contraloría General de la República al 23 de junio del 2020

El cuadro 1 muestra que, en la parte económica, para el departamento de La Libertad, se asignó un presupuesto de

a Johanna Jaritssielle, Zavaleta-Davis y ${ }^{\mathrm{b}}$ Leslye Anjy, Chávez-Santivañez Articulo Protegido por Licencia Creative Commons: BY-NC-ND / Protected by Creative Commons: BY-NC-ND. Sendas es una revista de acceso abierto / Sendas is an Open Access Journal. 
15.104.096 soles el cual repartido según los índices de población que poseía cada municipio donde las de menor población recibieron s/. 50,000.00 soles y los de más alta población un máximo de 2 millones de soles y bajo esta modalidad de reparto llegaron las cantidades señaladas en el cuadro $\mathrm{N}^{\circ} 1$, donde se detalla el avance presupuestal a nivel provincia donde se detalla el presupuesto, la cantidad de dinero habilitada y el dinero que vino siendo usado hasta el 20 de junio del 2020.

\begin{tabular}{|c|c|c|c|}
\hline \multicolumn{4}{|c|}{ Cuadro $N^{\circ} 1$} \\
\hline DEPARTAMENTO & $\begin{array}{l}\text { PRESUPUESTO } \\
\text { (PIM) }\end{array}$ & $\begin{array}{l}\text { CERTIFICADO } \\
\text { (HABIUTADO) }\end{array}$ & $\begin{array}{l}\text { CONTRATADC } \\
\text { (COMPROMISC }\end{array}$ \\
\hline JUNIN & 11.947 .482 & $11,934.387,51$ & 11.905 .4 \\
\hline E LA LIBERTAD & 15.104.096 & 14.962.282,43 & 14.781.6: \\
\hline ASCOPE & 1.227 .567 & $1.227 .565,98$ & 1.227 .5 \\
\hline BOLIVAR & 504,000 & $503.999,90$ & 503.9 \\
\hline CHEPEN & 606.300 & $606.299,36$ & 606.2 \\
\hline GRAN CHIMU & 641.775 & $626.737,30$ & 626.7 \\
\hline JULCAN & 757.260 & $748.987,66$ & 748.9 \\
\hline OTUZCO & 1.501 .830 & $1.501 .827,16$ & 1.501 .7 \\
\hline PACASMAYO & 902,400 & $902,400,00$ & 901.7 \\
\hline PATAZ & 1.808 .051 & $1.803 .051,00$ & 1.803 .0 \\
\hline SANCHEZ CARRION & 2.281 .122 & $2.176 .432,36$ & 2.010 .4 \\
\hline SANTIAGO DE CHUCO & 951.619 & $951.550,60$ & 951.5 \\
\hline TRUJILLO & 3.164 .395 & $3.155 .731,92$ & 3.144 .0 \\
\hline VIRU & 757.777 & $757.699,19$ & 755.5 \\
\hline I $\triangle M R \triangle Y F O U F$ & 7.054 .406 & 6.821 .125 .18 & 6.727 .3 \\
\hline
\end{tabular}

Fuente: Contraloría General de la República al 23 de junio del 2020

Se puede notar que las provincias más beneficiadas con el dinero son Trujillo, Sánchez Carrión Ascope, Otuzco y Pataz puesto que se les asigno un mayor presupuesto, por ende, debe interpretarse que tiene una mayor población, en el cuadro $\mathrm{N}^{\circ} 2$ siguiente se puede ver el avance donde solo Otuzco, Pataz y Trujillo tienen un avance donde más de la mitad de sus municipalidades han concluido con la distribución de las canastas.

Cuadro $N^{\circ} 2$

\begin{tabular}{l|rrr}
\multicolumn{1}{r|}{ DEPARTAMENTO } & CONCLUIDO & EN PROCESO & TOTAL \\
\hline LA LIBERTAD & 40 & 43 & 83 \\
ASCOPE & 4 & 4 & 8 \\
BOLIVAR & 5 & 1 & 6 \\
CHEPEN & 2 & 1 & 3 \\
GRAN CHIMU & 1 & 3 & 4 \\
JULCAN & & 4 & 4 \\
OTUZCO & 5 & 5 & 10 \\
PACASMAYO & 2 & 3 & 5 \\
PATAZ & 10 & 3 & 13 \\
SANCHEZ CARRION & 3 & 5 & 8 \\
SANTIAGO DE CHUCO & 2 & 6 & 8 \\
TRUILLLO & 6 & 5 & 11 \\
VIRU & & 3 & 3
\end{tabular}

Fuente: Contraloría General de la República al 23 de junio del 2020

La contraloría general de la republica realizo un control al Gobierno Regional de La Libertad el 17 de junio de 2020, un control concurrente donde se encontró deficiencias en el control de registro de operaciones de distribución de pruebas rápidas COVID - 19, tenían además un inadecuado almacenamiento y distribución de las pruebas rápidas puesto que se encontraron rodeados de bienes en desuso o siendo utilizados en estacionamiento de ambulancias.

a Johanna Jaritssielle, Zavaleta-Davis y ${ }^{\mathrm{b}}$ Leslye Anjy, Chávez-Santivañez Articulo Protegido por Licencia Creative Commons: BY-NC-ND / Protected by Creative Commons: BY-NC-ND Sendas es una revista de acceso abierto / Sendas is an Open Access Journal. 
La contraloría general de la republica realizo un control a las unidades ejecutoras de salud: región la libertad salud (Geresa- 11), instituto regional de oftalmología (Iro), Sánchez Carrión, Otuzco, Trujillo este (Utes 6) y Virú, del pliego Gobierno Regional La Libertad, al 4 de mayo de 2020 registran un avance de la ejecución presupuestal del 28,86\%, $18,49 \%, 15,04 \%, 12,55 \%, 28,31 \%$ у $16,98 \%$, respectivamente; revelando una baja capacidad de gasto, lo cual afectaría la adecuada y oportuna atención a los pacientes infectados y bajo sospecha de covid 19, además aumentaría el riesgo de contagio del personal asistencial.

\section{Grafica $N^{\circ} 6$}

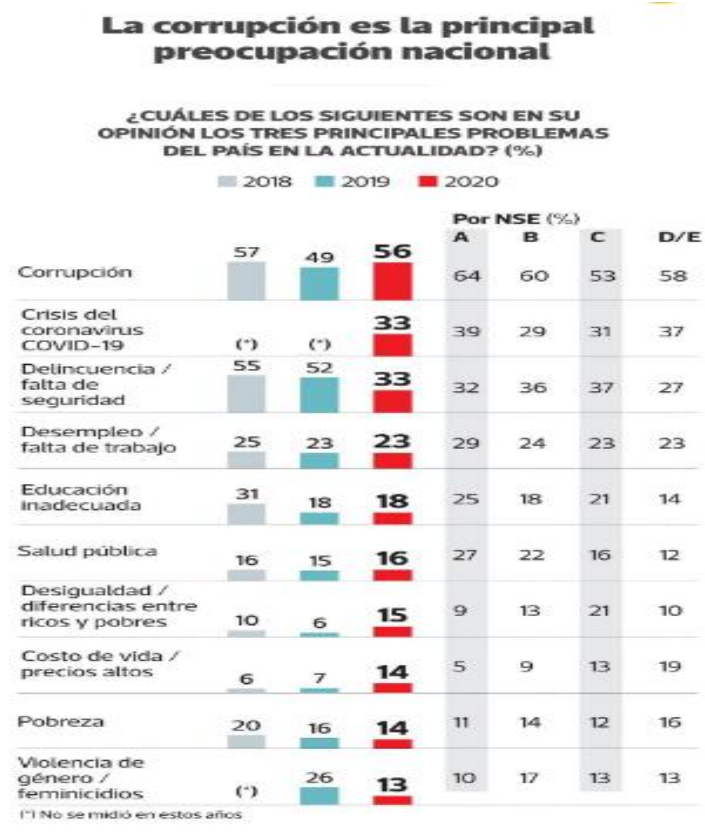

Fuente: El comercio-Ipsos 30de mayo del 2020

La encuestadora Ipsos Apoyo realizó una encuesta solicitando la opinión sobre los tres principales problemas que acogen al Perú, para medir el grado la percepción de los ciudadanos con respecto a la realidad nacional. Cuyos valores permiten observar que la corrupción $(56 \%)$ era la principal preocupación de los peruanos. Lo más sorprende aún es observar que se sobrepone a la emergencia sanitaria por covid-19(33\%), sin embargo, esta situación vendría liderando la preocupación de la población peruana

a Johanna Jaritssielle, Zavaleta-Davis y ${ }^{\mathrm{b}}$ Leslye Anjy, Chávez-Santivañez Articulo Protegido por Licencia Creative Commons: BY-NC-ND / Protected by Creative Commons: BY-NC-ND. Sendas es una revista de acceso abierto / Sendas is an Open Access Journal. 
desde el 2018. El segundo lugar sería para la delincuencia (33\%), que alcanzó el valor del mortal virus, y el tercero para el desempleo, que se mantuvo en un $23 \%$ con respecto al año anterior.

\section{Conclusiones}

Se determinó que pese a las transferencias económicas y los subsidios monetarios que suponen un apoyo para los sectores más golpeados por los efectos de la pandemia, no se ha logrado llegar ni a un $70 \%$ de avance de la ejecución del presupuesto asignado a las entidades públicas del gobierno regional y local de La Libertad, debido a la intervención de las irregularidades que cometen los "líderes "defensores del bienestar público, en el direccionamiento de los fondos para beneficio propio. Demostrando lo mucho que falta por madurar en la gestión pública aún retrógrada que se sostiene en un accionar egocéntrico, que ponen en riesgo la salud y el bienestar de la población.

En base a las cifras analizadas, se puede manifestar que la mayor inversión se realizó en el sector salud y en las compras de abastecimiento de primera necesidad. Sin embargo, una gran porción de los beneficiados fueron funcionarios públicos, quienes no formaban parte primordial de los destinatarios. Obstaculizando la llegada de los recursos a las poblaciones más vulnerables.

Que las malversaciones son reconocidas por los ciudadanos y conforman su principal preocupación sobreponiéndola incluso a los vicios sociales, comúnmente arraigados a la comunidad. Lo que supone a la vez una disconformidad con la gestión del gobierno, lo que podría enlentecer el proceso de recuperación de la crisis económica a causa de la emergencia sanitaria.

Que el bono canasta familiar fue el más fácil de identificar y en donde las personas notaron con mayor precisión la corrupción y esto se debió a que los bonos yo me quedo en casa, bono independiente, bono rural y el bono universal tenían no solo un registro de personas que lo necesitaban, sino también contaban con las

\footnotetext{
a Johanna Jaritssielle, Zavaleta-Davis y ${ }^{\mathrm{b}}$ Leslye Anjy, Chávez-Santivañez Articulo Protegido por Licencia Creative Commons: BY-NC-ND / Protected by Creative Commons: BY-NC-ND. Sendas es una revista de acceso abierto / Sendas is an Open Access Journal.
} 
trasferencias bancarias lo que al parecer hacia más difícil a los funcionarios públicos a la hora de coger estos fondos.

\section{$\underline{\text { Referencias }}$}

Baffes \&Nagle (23 de abril) Las perspectivas de los mercados de productos básicos y los efectos del coronavirus en seis gráficos. Banco Mundial Blogs. Recuperado de: https://blogs.worldbank.org/es/voic es/las-perspectivas-de-losmercados-de-productos-basicos-ylos-efectos-del-coronavirus-en-seis

Castro, J. (30 de abril del 2020). Encuesta El Comercio-Ipsos: La corrupción es el principal problema para los peruanos. Diario El Comercio. Recuperado:

https://elcomercio.pe/politica/actua lidad/encuesta-el-comercio-ipsosla-corrupcion-es-el-principalproblema-para-los-peruanosmartin-vizcarra-coronavirus-covid19-congreso-noticia/?ref $=e c r$
Conexión ESAN (24 de abril del 2020) Impacto del COVID-19 en el empleo en el Perú. Recuperado de: https://www.esan.edu.pe/conexion/a ctualidad/2020/04/24/impacto-delcovid-19-en-el-empleo-en-el-peru/

Contraloría general de la república del Perú (23 de junio del 2020). Ejecución presupuestal COVID-19: emergía sanitaria 2020. Recuperado:

https://emergenciasanitaria.contralo ria.gob.pe/ces_programacionejecucion-presup/index.html

Escalante, S. (04 de marzo del 2020) Corrupción y derechos humanos. Diario RRP Noticias. Recuperado de:

https://rpp.pe/columnistas/mariasol edadescalantebeltran/corrupcion-yderechos-humanos-noticia-1249243

Instituto Nacional de Salud -MINSA (23 de junio del 2020) Total de casos positivos por departamento: Sala situacional COVID-19 Perú. Recuperado de:

\footnotetext{
a Johanna Jaritssielle, Zavaleta-Davis y ${ }^{\mathrm{b}}$ Leslye Anjy, Chávez-Santivañez

Articulo Protegido por Licencia Creative Commons: BY-NC-ND / Protected by Creative Commons: BY-NC-ND.

Sendas es una revista de acceso abierto / Sendas is an Open Access Journal.
} 
https://covid19.minsa.gob.pe/sala_s ituacional.asp

Quiroz, A. (2014). Historia de la corrupción en el Perú. Edición popular. p. 486.

Montero, R. (11 de marzo del 2020) Dictan medidas urgentes $y$ excepcionales destinadas a reforzar el Sistema de Vigilancia y Respuesta Sanitaria frente al COVID-19 en el territorio nacional. Diario El Peruano. Recopilado de: https://busquedas.elperuano.pe/dow nload/url/dictan-medidas-urgentes-

y-excepcionales-destinadas-areforza-decreto-de-urgencia-n-0252020-1863981-1
Procuraduría Pública Especializada En Delitos De Corrupción. Ministerio de justicia y derechos humanos (junio de 2020) Boletín Informativo $N^{\circ} 01: \quad$ Corrupción En La Emergencia Sanitaria COVID19

Said, J. (2013) Corrupción administrativa, democracia y derechos humanos. Fórum Ltda. Brazil.

World Bank Group (Junio Del 2020) Global Economic Prospects. Recuperado de: https://www.bancomundial.org/e s/news/pressrelease/2020/06/08/covid-19-toplunge-global-economy-intoworst-recession-since-worldwar-ii\#

Corrupción en la gestión de recursos públicos asignados en la emergencia sanitaria COVID-19 en el gobierno regional de La Libertad - Perú 2020 (Johanna Jaritssielle Zavaleta-Davis) Por Revista Sendas se encuentra bajo una Licencia Creative Commons-No Comercial-Sin Derivadas 3.0 Uported.

a Johanna Jaritssielle, Zavaleta-Davis y ${ }^{\mathrm{b}}$ Leslye Anjy, Chávez-Santivañez Articulo Protegido por Licencia Creative Commons: BY-NC-ND / Protected by Creative Commons: BY-NC-ND. Sendas es una revista de acceso abierto / Sendas is an Open Access Journal. 